\title{
Numerical study of the cosmological velocity field as a function of density
}

\author{
A. Domínguez ${ }^{1,2}$ and A. L. Melott ${ }^{3}$ \\ ${ }^{1}$ Max-Planck-Institut für Metallforschung, Heisenbergstr. 3, 70569 Stuttgart, Germany \\ 2 Theoretische Physik, Ludwig-Maximilians-Universität, Theresienstr. 37, 80333 München, Germany \\ 3 Department of Physics and Astronomy, University of Kansas, Lawrence, Kansas 66045, USA
}

Received 23 October 2003 / Accepted 5 February 2004

\begin{abstract}
We report on a new study of the velocity distribution in $N$-body simulations. We investigate the center-of-mass and internal kinetic energies of coarsening cells as a function of time, cell size and cell mass. By using self-similar cosmological models, we are able to derive theoretical predictions for comparison and to assess the influence of finite-size and resolution effects. The most interesting result is the discovery of a polytropic-like relationship between the average velocity dispersion (internal kinetic energy) and the mass density in an intermediate range of densities, $\kappa_{\text {int }} \propto \varrho^{2-\eta}$. The exponent $\eta$ measures the deviations from the virial prediction, $\eta_{\text {virial }}=0$. For self-similar models, $\eta$ depends only on the spectral index of the initial power spectrum. We also study CDM models and confirm a previous result that the same polytropic-like dependence exists, with a time and coarsening length dependent $\eta$. The dependence $\kappa_{\text {int }}(\varrho)$ is an important input for a recently proposed theoretical model of cosmological structure formation which improves over the standard dust model (pressureless fluid) by regularizing the density singularities.
\end{abstract}

Key words. gravitation - methods: $N$-body simulations - cosmology: large-scale structure of Universe

\section{Introduction}

The use of $N$-body simulations has proved a useful tool in the investigation of both the cosmological structure formation and the evolution by self-gravity. The main interest has been concentrated on properties of the spatial distribution of matter (mass correlations, void distribution, morphological features...), while the kinetic properties have received comparatively little attention, without doubt due to the larger difficulties to obtain reliable kinetic measurements from real data with which to compare (Watkins et al. 2002).

The kinetic measurements addressed till now with $N$-body simulations have pertained the quasilinear velocity field (see e.g. the reviews by Dekel 1994 and Bernardeau et al. 2002; an application closely related to the present work is Seto \& Sugiyama 2001), the pairwise relative velocity (Peebles 1980; for recent applications, see e.g. Strauss et al. 1998; Feldman et al. 2003), and the velocity dispersion of halos (e.g. Knebe \& Müller 1999 in connection with the present work). Our work addresses the center-of-mass velocity of coarsening cells (macroscopic kinetic energy), as well as the velocity dispersion of the particles inside the cell (internal kinetic energy). The coarsening cells are randomly centered and of variable size

Send offprint requests to: A. Domínguez,

e-mail: alvaro@fluids.mpi-stuttgart.mpg.de (probing both the linear and the nonlinear regimes); in this way our analysis does not suffer the arbitrariness intrinsic to the definition of clusters and halos (Klypin \& Holtzmann 1997, and references therein), and essentially all the simulation particles are employed in the determination of the quantities. We are aware of two works where a similar analysis of $N$-body simulations has been performed (Kepner et al. 1997; Nagamine et al. 2001), motivated differently than ours. The connection to our work is explained in Sect. 5 in detail.

The present study focuses on the dependence of the cell kinetic energies on the cell mass density. The main motivation is the application to models of cosmological structure formation by self-gravity. The most widely used theoretical model is the dust model (pressureless fluid) (Peebles 1980; Padmanabhan 1995), which has been studied intensively (see e.g. the reviews Sahni \& Coles 1995; Bernardeau et al. 2002) but has the shortcoming of producing singularities. Some recent works (Buchert \& Domínguez 1998; Adler \& Buchert 1999; Buchert et al. 1999; Maartens et al. 1999; Domínguez 2000; Morita \& Tatekawa 2001; Domínguez 2002; Tatekawa et al. 2002) have proposed a novel approach. One of its features is the ability to derive adhesion-like models (Kofman \& Shandarin 1988; Gurbatov et al. 1989; Kofman et al. 1992; Melott et al. 1994; Sathyaprakash et al. 1995), and to offer a possible explanation of the physical origin for the "adhesive" behavior which 
regularizes the mass density singularities of the dust model. In these improved models, the internal kinetic energy brings about the "adhesive" behavior provided it can be approximated as a function of density and/or the gradients of the velocity field.

Another goal of this work is to confirm the results by Domínguez (1999, 2003), where a polytropic-like dependence between internal kinetic energy and mass density is found in $\mathrm{AP}^{3} \mathrm{M}$ simulations of CDM models. We indeed corroborate this finding in PM simulations of CDM models and also of selfsimilar models. The latter are particularly amenable to a theoretical analysis and allow the identification of the influence of finite-size and resolution effects in the measurements. We conclude that the polytropic-like dependence is unlikely to be an artifact of the simulations.

The paper is organized as follows: in Sect. 2 we work out the theoretical predictions for the density dependence of the macroscopic and internal kinetic energies. In Sect. 3 we describe the simulations and the method how we measure the kinetic energies. In Sect. 4 we present the results of the analysis. Section 5 contains a discussion of the results and the conclusions.

\section{Theoretical background}

Let $a(t)$ denote the cosmological expansion factor, $m$ the mass of a particle, and $\boldsymbol{x}_{\alpha}$ and $\boldsymbol{u}_{\alpha}$ the comoving position and peculiar velocity, respectively, of the $\alpha$ th particle. $W(\cdot)$ is a (normalized) smoothing window. Then, given a comoving smoothing scale $L$, the coarse-grained mass density field, velocity field and density of internal peculiar kinetic energy are defined respectively as follows:

$$
\begin{aligned}
& \varrho(\boldsymbol{x}, t ; L)=\frac{m}{[a(t) L]^{3}} \sum_{\alpha=1}^{N} W\left(\frac{\boldsymbol{x}-\boldsymbol{x}_{\alpha}(t)}{L}\right), \\
& \varrho \boldsymbol{u}(\boldsymbol{x}, t ; L)=\frac{m}{[a(t) L]^{3}} \sum_{\alpha=1}^{N} \boldsymbol{u}_{\alpha}(t) W\left(\frac{\boldsymbol{x}-\boldsymbol{x}_{\alpha}(t)}{L}\right),
\end{aligned}
$$

$K_{\mathrm{int}}(\boldsymbol{x}, t ; L)=\frac{m}{2[a(t) L]^{3}} \sum_{\alpha=1}^{N}\left|\boldsymbol{u}_{\alpha}(t)-\boldsymbol{u}(\boldsymbol{x}, t ; L)\right|^{2} W\left(\frac{\boldsymbol{x}-\boldsymbol{x}_{\alpha}(t)}{L}\right)$.

The density of macroscopic ${ }^{1}$ peculiar kinetic energy is defined as $K_{\mathrm{mac}}=(1 / 2) \varrho|\boldsymbol{u}|^{2}$, so that one can write $K_{\mathrm{tot}}=K_{\mathrm{int}}+K_{\mathrm{mac}}$, where $K_{\text {tot }}$ is the density of total peculiar kinetic energy: this is given by the same expression as $K_{\text {int }}$ above but dropping $\boldsymbol{u}$. Whether $K_{\text {mac }}$ or $K_{\text {int }}$ dominates the contribution to $K_{\text {tot }}$ means respectively that the particle velocities $\boldsymbol{u}_{\alpha}$ are controlled by the center-of-mass motion of the coarsening cell as a whole or by "internal" motions within the cell (the ratio $K_{\text {mac }} / K_{\text {int }}$ was introduced as the cosmic Mach number by Ostriker \& Suto 1990). Indeed, $K_{\mathrm{int}}$ is a much better probe of the dynamics at the small scales than $K_{\text {mac }}$, because in the latter there can be extensive cancellations in the vectorial sum defining $\boldsymbol{u}$.

\footnotetext{
${ }^{1}$ Borrowing the nomenclature from statistical physics.
}

The purpose of the present study is the relationship between $K_{\text {int }}$ and the field $\varrho$. Our main interest is the density dependence of $\left\langle K_{\text {int }}\right\rangle_{\varrho}$, that is, the average of $K_{\text {int }}$ conditioned to a given value $\varrho$ of the coarse-grained mass density,

$\kappa_{\text {int }}(\varrho, t ; L):=\left\langle K_{\text {int }}\right\rangle_{\varrho}=\frac{\left\langle K_{\text {int }}(\boldsymbol{x}, t ; L) \delta_{\mathrm{D}}(\varrho(\boldsymbol{x}, t ; L)-\varrho)\right\rangle}{\left\langle\delta_{\mathrm{D}}(\varrho(\boldsymbol{x}, t ; L)-\varrho)\right\rangle}$,

where $\langle\cdot\rangle$ denotes ensemble average, which by translational invariance must be $\boldsymbol{x}$-independent, and $\delta_{\mathrm{D}}(\cdot)$ is Dirac's delta function. In the same way is defined the conditioned average $\kappa_{\text {mac }}:=\left\langle K_{\text {mac }}\right\rangle_{\varrho}$. Since $\kappa_{\text {int }}$ receives contributions from the highly non-linear regime, the density dependence cannot be computed analytically in general. Nevertheless, a lot can be learned by way of suitable approximations, whose validity will be checked by comparing with simulations.

To simplify the theoretical discussion, we consider a selfsimilar cosmological model: an Einstein-de Sitter background and an initial Gaussian distributed density field with power spectrum $P\left(k, t_{\text {initial }}\right)=A k^{n}$, with the bounds $n>-3$ (so that density fluctuations do not receive a divergent contribution from $k \rightarrow 0$ ) and $n<4$ (imposed by the unavoidable graininess due to the point particles) (Peebles 1980; Padmanabhan 1995). The conclusions should apply qualitatively unaltered to a more realistic case. Let $\sigma^{2}(L, t)=\left\langle\delta^{2}(\boldsymbol{x}, t ; L)\right\rangle$ denote the variance of the density contrast smoothed on the scale $L\left(\delta:=\left(\varrho / \varrho_{b}\right)-1\right.$ is the density contrast, with $\varrho_{b}:=\langle\varrho\rangle$ the background density),

$\sigma^{2}(L, t)=\int \frac{\mathrm{d} \boldsymbol{k}}{(2 \pi)^{3}} P(k, t)|\tilde{W}(L \boldsymbol{k})|^{2}$.

(A tilde will denote a Fourier transform.) A (comoving) scale of nonlinearity, $r_{\mathrm{nl}}(t)$, is defined by the condition

$\int_{k<2 \pi / r_{\mathrm{nl}}} \mathrm{d} \boldsymbol{k} P(k, t)=1$.

In the self-similar model, the physical properties which do not depend on the short and large distance cutoffs of $P(k)$ exhibit a simple scaling behavior (Peebles 1980; Padmanabhan 1995).

The only physically relevant parameters on which they can depend are $\varrho_{b}\left(t_{0}\right)$ (through the cosmological background), $a_{\mathrm{nl}}\left(t_{0}\right)$ (through the initial conditions), and the gravitational constant $G$ (through the dynamics); self-similarity means that the time $t_{0}$ is arbitrary. Thus, in combination with dimensional analysis ${ }^{2}$, it is shown that $\sigma(L, t)$ is a function of the single quantity $L / r_{\mathrm{nl}}(t)$; in the linear regime, $L \gg r_{\mathrm{nl}}$, one has $\sigma^{2} \propto\left(L / r_{\mathrm{nl}}\right)^{-n-3}$. Similarly, $\kappa_{\text {int }} /$ mac can be written in the following suggestive form:

$\kappa_{\mathrm{int} / \mathrm{mac}}(\varrho, t ; L)=\varrho_{b}\left(\dot{a} r_{\mathrm{nl}}\right)^{2} F_{\mathrm{int} / \mathrm{mac}}\left(\delta, \frac{L}{r_{\mathrm{nl}}}\right)$.

Here $F(\cdot)$ is a dimensionless function of its dimensionless arguments, and $\varrho_{b}\left(\dot{a} r_{\mathrm{nl}}\right)^{2}$ is of the order of the Hubble-flow kinetic energy in balls of (comoving) radius $r_{\mathrm{nl}}$. The unconditioned averages $\left\langle\kappa_{\text {int }}\right\rangle=\left\langle K_{\text {int }}\right\rangle$ and $\left\langle K_{\text {mac }}\right\rangle$ follow the same scaling but without the $\delta$-dependence. Deviations from this scaling behavior would mean a dependence on extra variables, e.g., on short

\footnotetext{
${ }^{2}$ Here it is to be distinguished between the dimensions of comoving and physical lengths, differing by a factor $a(t)$.
} 


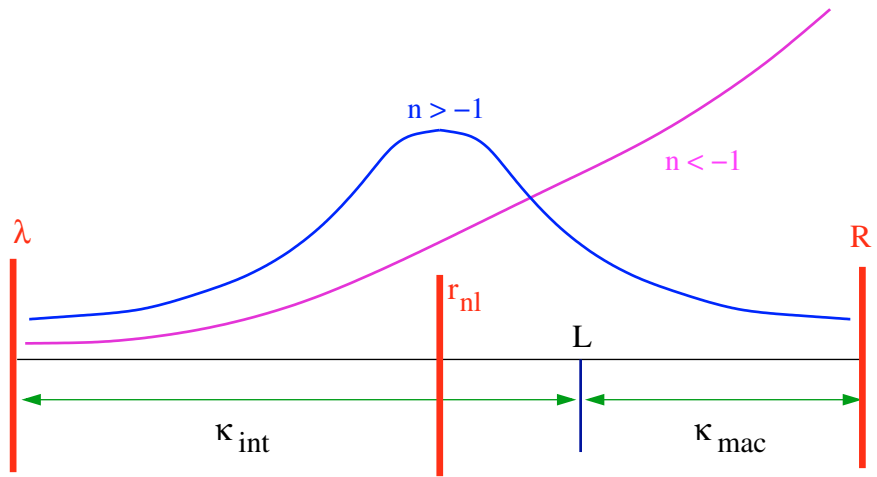

Fig. 1. Sketch representing the relative contribution of the different length scales to $\kappa_{\mathrm{mac}}$ and $\kappa_{\mathrm{int}}$. The scales $\lambda, R$ and $r_{\mathrm{nl}}$ are intrinsic to the system, the movable length $L$ is the observation resolution.

or large distance cutoffs in $P(k)$ or in the dynamics (as occurs with, e.g., numerical simulations).

The task now is to characterize the functions $F_{\text {int } / \text { mac }}(\cdot)$. Let $\lambda$ and $R$ denote the short and the large distance cutoffs, respectively, so that we take $\lambda \ll r_{\mathrm{n}}, L \ll R$. Roughly speaking, we can say that $\kappa_{\mathrm{mac}}$ is determined by the motion at scales between $L$ and $R$, whereas $\kappa_{\mathrm{int}}$ is dominated by the motion at scales between $\lambda$ and $L$.

The hierarchical, bottom-up scenario exhibits a monotonically growing length scale, $r_{\mathrm{nl}}(t)$, which is roughly proportional to the size of the largest collapsed clusters at time $t$. The bottom-up growth of structure by self-gravity can be sketched in the following picture: particles get trapped in clusters so that (i) the evolution above the cluster scale is dominantly ruled by the motion of each cluster as a whole (="effective particles") in the gravitational field of the other clusters, and (ii) the evolution below the cluster scale is driven mainly by the scales $\sim$ cluster size. This means a dynamical decoupling between scales above and below the cluster size, $\sim r_{\mathrm{nl}}$ (this idea has been explored by Domínguez $(2000,2002)$, in order to improve the models of structure formation), and implies that the short-distance cutoff $\lambda$ is irrelevant. Depending on which of the two motions, (i) or (ii), contributes mostly to the particle velocity, there arise several possibilities (see Fig. 1):

- if $-3<n<-1$, it will be argued that the largest scales dominate. Thus, $\kappa_{\text {mac }}$ should be determined by the scales $\sim R$ and $\kappa_{\text {int }}$ by the scales $\sim L$;

- if $-1<n<4$, the scales around $r_{\mathrm{nl}}$ provide the prevalent contribution because $\lambda$ is irrelevant and there is no other privileged scale between $\lambda$ and $r_{\mathrm{nl}}$. We can distinguish in turn two cases:

- linear regime, $r_{\mathrm{nl}} \ll L$ : the value of $\kappa_{\text {mac }}$ is mainly set by scales $\sim L$, and that of $\kappa_{\text {int }}$ by scales $\sim r_{\mathrm{nl}}$;

- nonlinear regime, $L \ll r_{\mathrm{nl}}$ : now $\kappa_{\mathrm{mac}}$ is dominated by scales $\sim r_{\text {nl }}$, and $\kappa_{\text {int }}$ by scales $\sim L$.

This description will be now elaborated in somewhat more detail.
Case I: $-3<n<-1$. There is so much power initially at the large scales, that the contribution of the linear modes $\left(r_{\mathrm{nl}} k \ll 1\right)$ to the variance of the macroscopic velocity diverges if $n<-1$ :

$\left\langle|\boldsymbol{u}|^{2}\right\rangle \propto \int_{0}^{r_{\mathrm{n} 1}^{-1}} \mathrm{~d} \boldsymbol{k} k^{n-2}+$ nonlinear contribution.

Hence, both $\boldsymbol{u}$ and the particle velocities $\boldsymbol{u}_{\alpha}$ will be mainly determined by the modes around the largest available scale, the infrared (IR) cutoff $R$. For the macroscopic kinetic energy we then estimate

$\kappa_{\text {mac }} \sim \varrho\left\langle|\boldsymbol{u}|^{2}\right\rangle_{\varrho} \sim \varrho_{b}(\dot{a} R)^{2} \sigma^{2}(R)(1+\delta)$,

$\left\langle K_{\mathrm{mac}}\right\rangle \sim \varrho_{b}(\dot{a} R)^{2} \sigma^{2}(R)$.

For the internal kinetic energy, it proves useful to consider separately the linear and nonlinear regimes:

- Linear regime, $L \gg r_{\mathrm{nl}}$. Our hypothesis is that the main contribution to $\kappa_{\text {int }}$ comes from scales $\sim L$, so that we can employ the linear solutions to the gravitational instability to evaluate Eqs. (1). The calculations are collected in the Appendix; the final result reads

$\kappa_{\text {int }}=\left\langle K_{\text {int }}\right\rangle\left[1+B\left(1-\frac{\delta^{2}}{\sigma^{2}(L)}\right)\right]$.

In this expression, $\left\langle K_{\text {int }}\right\rangle$ is the unconditioned average internal peculiar kinetic energy in the linear approximation, Eq. (A.2), $B$ is a dimensionless negative numerical coefficient, Eq. (A.3). Both $\left\langle K_{\text {int }}\right\rangle$ and $B$ are IR-convergent because the $R$-dependence cancels from the difference $\boldsymbol{u}_{\alpha}-\boldsymbol{u}$, so that Eq. (3) holds. Indeed, we derive the scaling behavior

$\left\langle K_{\text {int }}\right\rangle \propto \varrho_{b}(\dot{a} L)^{2} \sigma^{2}(L)$.

- Nonlinear regime, $L \ll r_{\mathrm{nl}}$. In high-density coarsening cells, $\delta \gg 1$, the Hubble flow is negligible and peculiar velocities are approximately equal to the physical velocities. Assuming stationarity, the conditioned differences $\left\langle\left|\mathbf{u}_{\alpha}-\boldsymbol{u}\right|^{2}\right\rangle_{\varrho}$ can be expected to be given by local virialization on scales $\sim L$, so that

$\kappa_{\mathrm{int}} \sim \frac{1}{(a L)^{3}} \frac{G\left[\varrho(a L)^{3}\right]^{2}}{a L} \sim \varrho_{b}(\dot{a} L)^{2} \delta^{2}, \quad(\delta \gg 1)$.

The opposite limit of low density, $0<1+\delta \ll 1$, requires a model for the expansion of voids. The simplest model would set $\left|\boldsymbol{u}_{\alpha}-\boldsymbol{u}\right| \sim h L$ typically, with $h \approx \dot{a}$ quantifying the void expansion speed. Then

$\kappa_{\text {int }} \sim \varrho_{b}(\dot{a} L)^{2}(1+\delta), \quad(0<1+\delta \ll 1)$,

predicting a low internal kinetic energy.

To compute the unconditioned average $\left\langle K_{\text {int }}\right\rangle$, it must be noticed that, although the internal kinetic energy of highdensity cells is very large, the number of low-density cells is much larger, and it is not clear which of the two competing effects dominates. If one would assume that the main contribution comes from high-density cells, then Eq. (8) would yield

$\left\langle K_{\text {int }}\right\rangle \sim \varrho_{b}(\dot{a} L)^{2} \sigma^{2}(L)$. 
Case II: $-1<n<4$. The linear modes do not lead to divergences and the velocities are now determined mainly by the scale $r_{\mathrm{nl}}$.

- Linear regime, $L \gg r_{\mathrm{nl}}$. Now the linear solution states that the smoothed velocity $\boldsymbol{u}$ will be determined by scales $\sim L$, i.e., the smallest scales it probes, and thus one obtains

$\kappa_{\mathrm{mac}} \sim \varrho_{b}\left\langle|\boldsymbol{u}|^{2}\right\rangle \sim \varrho_{b}(\dot{a} L)^{2} \sigma^{2}(L), \quad\left\langle K_{\mathrm{mac}}\right\rangle \sim \kappa_{\mathrm{mac}}$.

On the other hand, our hypothesis is that the particle velocities $\boldsymbol{u}_{\alpha}$ are controlled by scales $\sim r_{\mathrm{nl}}$. Hence, we write the estimate

$\left\langle\left|\boldsymbol{u}_{\alpha}\right|^{2}\right\rangle_{\varrho} \sim \frac{G \varrho_{b}\left(a r_{\mathrm{nl}}\right)^{3}}{a r_{\mathrm{nl}}} \sim\left(\dot{a} r_{\mathrm{nl}}\right)^{2}$,

assuming that the scales $\sim r_{\mathrm{nl}}$ are rather insensitive to the small density fluctuations at the scale $L \gg r_{\mathrm{nl}}$. Then

$\kappa_{\mathrm{int}} \sim \varrho_{b}\left(\dot{a} r_{\mathrm{nl}}\right)^{2}(1+\delta), \quad\left\langle K_{\mathrm{int}}\right\rangle \sim \varrho_{b}\left(\dot{a} r_{\mathrm{nl}}\right)^{2}$.

- Nonlinear regime, $L \ll r_{\mathrm{nl}}$. The conditioned difference $\left\langle\left|\boldsymbol{u}_{\alpha}-\boldsymbol{u}\right|^{2}\right\rangle_{\varrho}$ is controlled by the local dynamics on scales $\sim L$ like in the previous case, and expressions (8-10) are valid now too. The motion of the coarsening cells as a whole, and thus $\left\langle|\boldsymbol{u}|^{2}\right\rangle_{\varrho}$, is however assumed to be determined by the large scales $\sim r_{\mathrm{n}}$, so that

$\kappa_{\mathrm{mac}} \sim \varrho_{b}\left(\dot{a} r_{\mathrm{nl}}\right)^{2}(1+\delta), \quad\left\langle K_{\mathrm{mac}}\right\rangle \sim \varrho_{b}\left(\dot{a} r_{\mathrm{nl}}\right)^{2}$,

in analogy to Eqs. (12).

\section{Simulation and analysis method}

The simulations of the self-similar models are described in full detail elsewhere (Melott \& Shandarin 1993). They consist of a cubic box (periodic boundary conditions) of comoving sidelength $R$ containing $N=128^{3}$ particles. The dynamical evolution was computed using a PM algorithm on a grid with Nyquist wavenumber $=128 \pi / R$. The background cosmological expansion followed the Einstein-de Sitter solution and the initial conditions were generated by using the Zel'dovich approximation for a Gaussian random field with a scale-invariant power spectrum $P(k) \propto k^{n}$. In the present work, the values $n=-2,0,+1$ were considered and the data were studied at three different times, corresponding to a scale of nonlinearity $r_{\mathrm{nl}}=R / 64, r_{\mathrm{nl}}=R / 16$, and $r_{\mathrm{nl}}=R / 4$, respectively. In each case four independent realizations of the initial conditions were evolved.

Two CDM models were also addressed with a PM algorithm: flat $\operatorname{CDM}\left(\Omega_{m}=1.0, \Omega_{\Lambda}=0.0, \sigma_{8} \approx 1\right)$, and open $\operatorname{CDM}\left(\Omega_{m}=0.3, \Omega_{\Lambda}=0.0, \sigma_{8} \approx 1\right)$. Each simulation contained $N=256^{3}$, and for each model two different boxsizes were considered, $R=128 \mathrm{Mpc}$ and $R=512 \mathrm{Mpc}$.

Starting from the coordinates $\left\{\boldsymbol{x}_{\alpha}, \boldsymbol{u}_{\alpha}\right\}_{\alpha=1 \ldots N}$ provided by the simulation, the definitions (1) were applied with a cubic top-hat window,

$W(z)=\theta\left(1-2\left|z_{1}\right|\right) \theta\left(1-2\left|z_{2}\right|\right) \theta\left(1-2\left|z_{3}\right|\right)$,

where $\theta(\cdot)$ is the step function. The coarsening was implemented efficiently by covering the simulation box with a cubic grid; in order to probe a minimum of 2048 coarsening cells, the grid was shifted randomly, when needed. It was checked that the results are insensitive to the use of a spherical top-hat window instead. A comparison with the use of a Gaussian window was also carried out. We observed a difference only for the case $n=+1$ at the earliest time, $r_{\mathrm{nl}}=R / 64$, because then $\sigma^{2}$ diverges for a top-hat window (in the simulation, the artificial point-particle discreteness regularizes the singularity). Hence, for this single case we disregarded the use of the cubic tophat window as unphysical and used instead a Gaussian window (which is computationally much more costly).

The explored values of the coarsening length $L$ were equally separated in a logarithmic scale and they ranged from a maximum $(R / 5)$ down to a minimum $\approx \ell / 2$, where $\ell:=$ $R / N^{1 / 3}$ is the average interparticle distance. This results in scatter plots "kinetic energy vs. density". To compute the constrained average (2), the data for the kinetic energy were binned into 40 subintervals according to the value of the density $\varrho$; bins containing less than 10 data points were disregarded. It was checked that the conclusions are robust against the amount of binning by varying the number of bins. The constrained average was identified with the mean of each bin. The amount of scatter about this (global) mean is represented in the plots by scatter bars, which extend between the mean of those kinetic energies above the global mean, and the mean of those kinetic energies below the global mean. We find that this method represents the scatter of the data in the log-plots more faithfully than the estimation through the variance.

We checked the algorithm in various ways. It was applied to an ideal gas simulation: the results for the dependence of $\kappa_{\mathrm{int}}$ on the density agreed with the ideal gas equation of state. Another check was to restrict the coarsening procedure to a subvolume of the simulation box (1/64 of the total volume) for some sample cases. As expected, we find that the data are somewhat noisier because of the reduced number of particles, but the conclusions remain the same.

\section{Results}

There are some general remarks which hold for all the subsections to follow. First, the small length scale $\ell$ enters in the results via mass-resolution and force-resolution effects: the first effect refers to the presence of a minimum non-vanishing mass - that of a single particle. This affects the computation of the unconstrained averages $\left\langle K_{\mathrm{int} / \mathrm{mac}}\right\rangle$ due to undersampling of the cells with a mass smaller than this minimum, which also sets a lower bound on the value of the density at a given fixed $L$ when computing the constrained averages, $\kappa_{\mathrm{int}}, \kappa_{\mathrm{mac}}$. In particular, it renders all results concerning the nonlinear regime $\left(L<r_{\mathrm{nl}}\right)$ at the earliest probed time $\left(r_{\mathrm{nl}}=R / 64\right)$ rather unreliable, since then $r_{\mathrm{nl}} \approx \ell$. The second effect, force resolution, implies that the relative force over two particles decreases when they are closer than the mesh spacing of the PM algorithm, $\approx \ell$, and the velocity dispersion below this scale does not grow as much as it would if $\ell \rightarrow 0$. All in all, these two effects tend to artificially reduce the value of the kinetic energy, in particular of $K_{\text {int }}$, being more sensitive to the small scales. The theoretical discussion in Sect. 2 suggests this effect to be particularly 
noticeable when $n>-1$ and at the earliest times, as indeed will be observed.

Second, the influence of the "cosmic variance", i.e., of the fluctuations in measured quantities from one realization to another, is the strongest when $n=-2$. For clarity, however, we will show in the plots the results of a single realization, since the other ones yield almost identical results.

\subsection{Self-similar models: Mass density variance}

For reference purposes, Fig. 2 shows the measurements of $\sigma^{2}(L)$. The results collapse well on a single function of $L / r_{\mathrm{nl}}$. At the earliest time and the smallest lengths, one can observe the beginning of the crossover to the Poissonian behavior, $\sigma^{2}(L) \propto L^{-3}$, induced by the small-scale discreteness. At large $L$, one recovers the linear scaling, $\sigma^{2}(L) \propto L^{-n-3}$; due to finite-size effects, the case $n=-2$ exhibits a slight departure away from this dependence.

\subsection{Self-similar models: The unconstrained averages}

The average $\left\langle K_{\text {mac }}\right\rangle$ is the kinetic equivalent of $\sigma^{2}$. In Fig. 3, we observe that the data for $\left\langle K_{\mathrm{mac}}\right\rangle$ do not follow at all the scaling behavior (3) when $n=-2$. As explained in Sect. 2, this is due to finite-size effects: we have checked that the data follow instead the dependence (5). The data for the other two cases, $n=0,+1$ on the contrary, obey the expected scaling, Eq. (11) when $L \gg r_{\mathrm{nl}}$, and Eq. (13) when $L \ll r_{\mathrm{nl}}$. A major departure in the three cases is observed at the earliest time $\left(r_{\mathrm{nl}}=R / 64\right)$ in the nonlinear regime $\left(L<r_{\mathrm{nl}}\right)$ due to the undersampling problem mentioned above. This was confirmed by artificially removing from the estimate of the averages those cells with less than a given number of particles, yielding the same behavior in $\left\langle K_{\mathrm{mac}}\right\rangle$ as detected in the plots.

As remarked in Sect. 2, the average $\left\langle K_{\text {int }}\right\rangle$ is more sensitive to the small-scale dynamics than $\left\langle K_{\mathrm{mac}}\right\rangle$ or $\sigma^{2}$ are. This average also suffers the same undersampling problem as $\left\langle K_{\mathrm{mac}}\right\rangle$. But the resolution effects are somewhat larger and prevent the data from following the scaling (3) perfectly. One can recognize a tendency for these effects to become less relevant in time, and to be more important for larger values of the spectral index $n$, in agreement with the theoretical discussion.

\subsection{Self-similar models: The constrained averages}

\subsubsection{Check for self-similarity}

We first checked if the measured $\kappa_{\text {int/mac }}$ followed the selfsimilar scaling relationship (3). The conclusions are almost the same as derived above with the unconstrained averages $\left\langle K_{\text {int } / \text { mac }}\right\rangle: \kappa_{\text {mac }}$ follows self-similarity very well (except if $n=-2$ ), while $\kappa_{\text {int }}$ follows it a bit less well. The important difference is that departures from self-similarity are (sometimes substantially) smaller than in $\left\langle K_{\text {int } / \text { mac }}\right\rangle$, see Fig. 4 . The reason is that $\kappa_{\mathrm{int} / \mathrm{mac}}$ suffer the undersampling problem due to finite mass-resolution only in the small- $\delta$ end of each curve or at the earliest time. When the number of particles in the cell is large
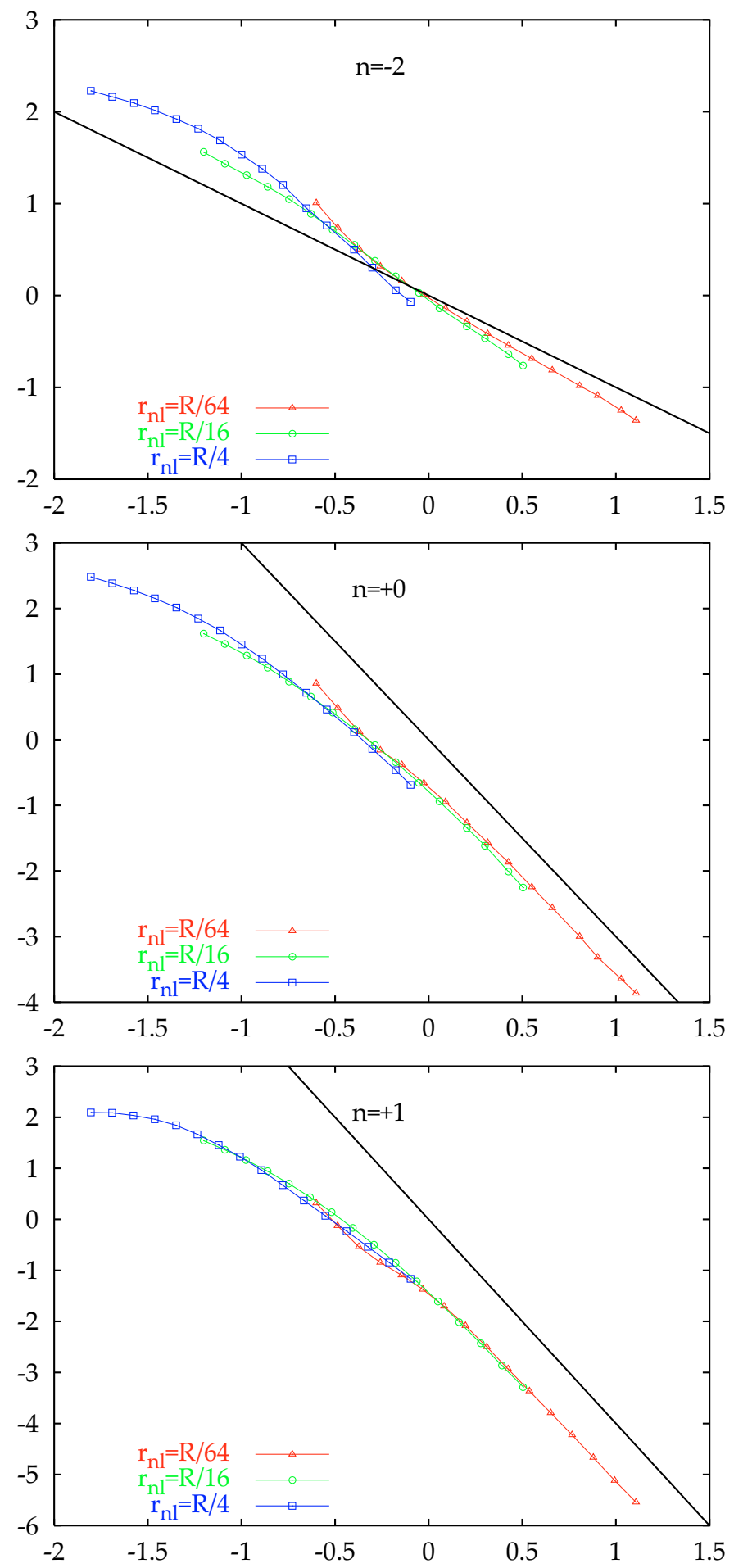

Fig. 2. Abscissa: $\log \left(L / r_{\mathrm{nl}}\right)$. Ordinate: $\log \sigma^{2}(L)$ at the three probed times. Solid line: expected functional dependence in the linear regime, $\sigma^{2} \propto L^{-n-3}$.

enough, force-resolution is likely the main effect and it does not appear to spoil self-similarity so much.

\subsection{2. $\kappa_{\mathrm{mac}}$}

Figure 5 shows the function $\kappa_{\mathrm{mac}}(\delta)$ at the different times and coarsening lengths probed for the spectral index $n=-2$. The scaling Eq. (4) is obeyed well at all times, although we find 

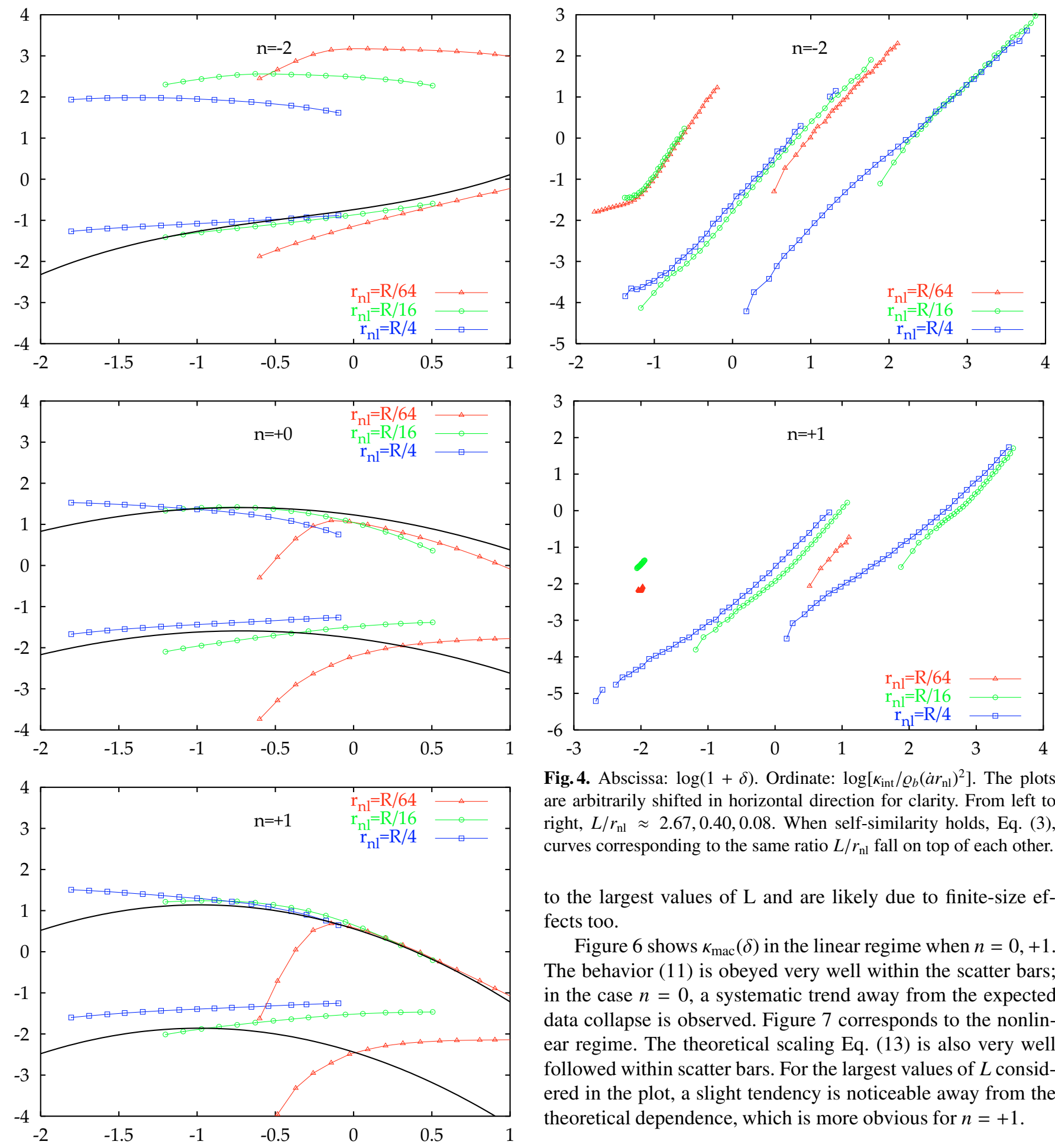

Fig. 4. Abscissa: $\log (1+\delta)$. Ordinate: $\log \left[\kappa_{\mathrm{int}} / \varrho_{b}\left(\dot{a} r_{\mathrm{nl}}\right)^{2}\right]$. The plots are arbitrarily shifted in horizontal direction for clarity. From left to right, $L / r_{\mathrm{nl}} \approx 2.67,0.40,0.08$. When self-similarity holds, Eq. (3), curves corresponding to the same ratio $L / r_{\mathrm{nl}}$ fall on top of each other.

to the largest values of $\mathrm{L}$ and are likely due to finite-size effects too.

Figure 6 shows $\kappa_{\text {mac }}(\delta)$ in the linear regime when $n=0,+1$. The behavior (11) is obeyed very well within the scatter bars; in the case $n=0$, a systematic trend away from the expected data collapse is observed. Figure 7 corresponds to the nonlinear regime. The theoretical scaling Eq. (13) is also very well followed within scatter bars. For the largest values of $L$ considered in the plot, a slight tendency is noticeable away from the theoretical dependence, which is more obvious for $n=+1$.

Fig. 3. Abscissa: $\log \left(L / r_{\mathrm{nl}}\right)$. Ordinate: $\log \left[\langle K\rangle / \varrho_{b}\left(\dot{a} r_{\mathrm{nl}}\right)^{2}\right]$ at the three probed times: the lower points in each plot correspond to $\left\langle K_{\text {int }}\right\rangle$; the upper points represent $10^{3}\left\langle K_{\mathrm{mac}}\right\rangle$. The solid line is proportional to $L^{2} \sigma^{2}(L)$, Eqs. (7), (10), (11).

small fluctuations around the linear dependence between realizations. This is due to the strong dependence on the IR cutoff. It is also responsible for the lack of collapse of the three plots on a single function, the deviations following indeed the law $R^{2} \sigma^{2}(R)$ of the factor in Eq. (4). The slight deviations around $\delta=0$, most noticeable at the earliest time, correspond

\subsection{3. $\kappa_{\text {int }}$, linear regime}

Figure 8 shows $\kappa_{\text {int }}(\delta)$ for $n=-2$ in the linear regime. Deviations from the theoretical prediction, Eq. (6) with $\left\langle K_{\text {int }}\right\rangle$ and $B$ computed with Eqs. (A.2), (A.3), are noticeable. The probable reason for the discrepancy is that the linear regime is not really being probed: one can observe a large asymmetry $\delta \rightarrow-\delta$ in the plot, in contradiction with a centered Gaussian distribution for $\delta$. (That deviations from Gaussianity are indeed important has been checked by estimating the probability distribution of $\delta$ from the simulations.) The most 


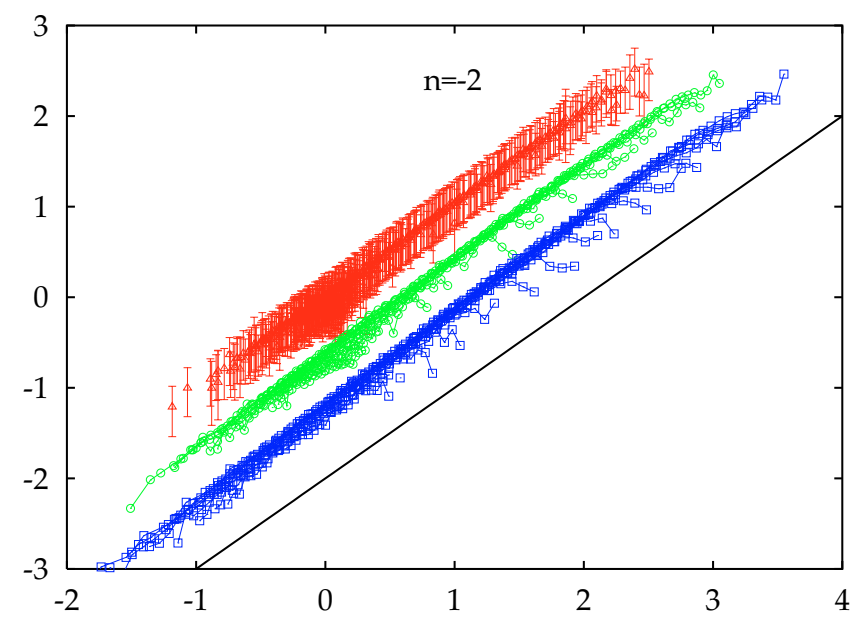

Fig. 5. Abscissa: $\log (1+\delta)$. Ordinate: $\log \left[\kappa_{\mathrm{mac}} / \varrho_{b}\left(\dot{a} r_{\mathrm{nl}}\right)^{2}\right]$. Solid line: theoretical functional dependence, Eq. (4). All the probed coarsening lengths, at the three probed times (increasing from top to bottom) are plotted. The scatter bars are barely dependent on time, coarsening length and density; for clarity, they are shown only at the earliest time.

linear case we probe for $n=-2$ (corresponding to $r_{\mathrm{nl}}=R / 64$ and $L=R / 5$ ) yields $\sigma \approx 0.2$, which satisfies the asymptotic condition $\sigma \ll 1$ only marginally. Thus, the ultimate origin of the problem is the IR cutoff imposed by the simulation box which limits the maximum value of $L$.

Figure 9 represents $\kappa_{\text {int }}(\delta)$ when $n=0,+1$. According to Eq. (12), $\kappa_{\text {int }}$ is determined by the velocities at scales $r_{\mathrm{nl}}$, which is $\sim \ell$ at the earliest time (corresponding to the plotted data). Thus, the results are not too reliable in principle, as discussed above. Indeed, the collapse of the data on a master curve is marginal within the scatter bars, $\kappa_{\text {int }}$ exhibits a trend to decrease with decreasing coarsening length - consistent with the artificial reduction of kinetic energy at small scales by resolution effects. The linear dependence predicted by Eq. (12) is not observed, a curvature being evident. But we cannot conclude if this is due to resolution effects or because the derivation of Eq. (12) relies on too simple arguments.

\subsection{4. $\kappa_{\text {int }}$, nonlinear regime}

Figure 10 shows $\kappa_{\text {int }}$ in the nonlinear regime at the latest time, $r_{\mathrm{nl}}=R / 4$. For each coarsening length, the theoretical functional dependence, Eq. (8), is obeyed only at the largest densities, if at all. At intermediate densities ${ }^{3}$, Fig. 11 shows that the data can be better fitted by the following polytropiclike behavior:

$\kappa_{\text {int }} \sim \varrho_{b}(\dot{a} L)^{2} \sigma^{2}(L) \delta^{2-\eta}$,

where the exponent $\eta$ is detectably different from the virial prediction $(\eta=0)$. A fit by eye yields the rough values $\eta(n=$ $-2) \approx 0.3, \eta(n=0) \approx 0.5, \eta(n=+1) \approx 0.6-$ the amount of scatter does not allow a more precise determination of $\eta$; if the scatter is forgotten, it could even be that $\eta$ depends slightly on $L$, as seems to be the case when $n=-2$, Fig. 10 .

\footnotetext{
3 The data tend to follow the fit also for very small densities as long as mass-resolution effects are not large.
}
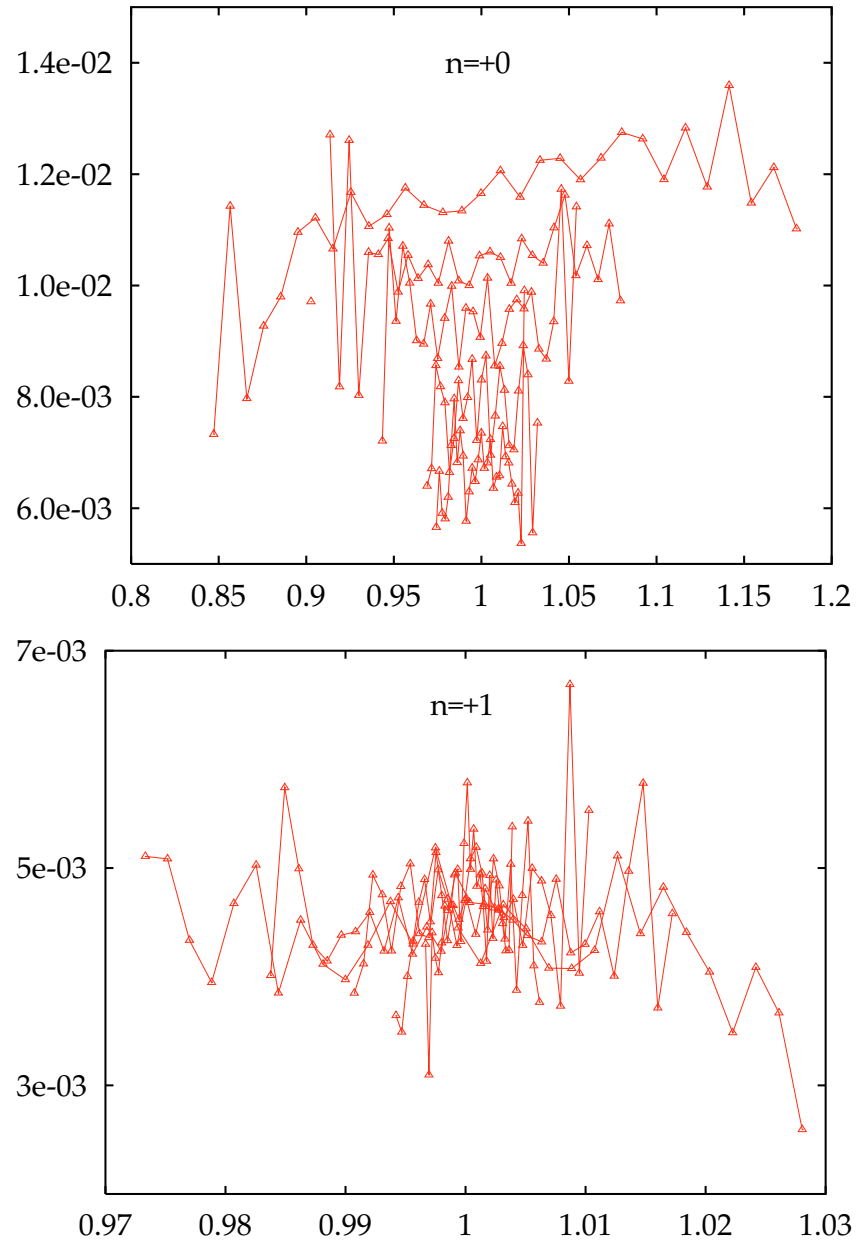

Fig. 6. Abscissa: $1+\delta$. Ordinate: $\kappa_{\mathrm{mac}} / \varrho_{b}[\dot{a} L \sigma(L)]^{2}$. Only plotted are the coarsening lengths such that $\sigma(L)<0.05$ if $n=0$ and $\sigma(L)<$ 0.03 if $n=+1$. The theoretical functional dependence, Eq. (11), is a constant. The scatter bars extend beyond the plotted area.

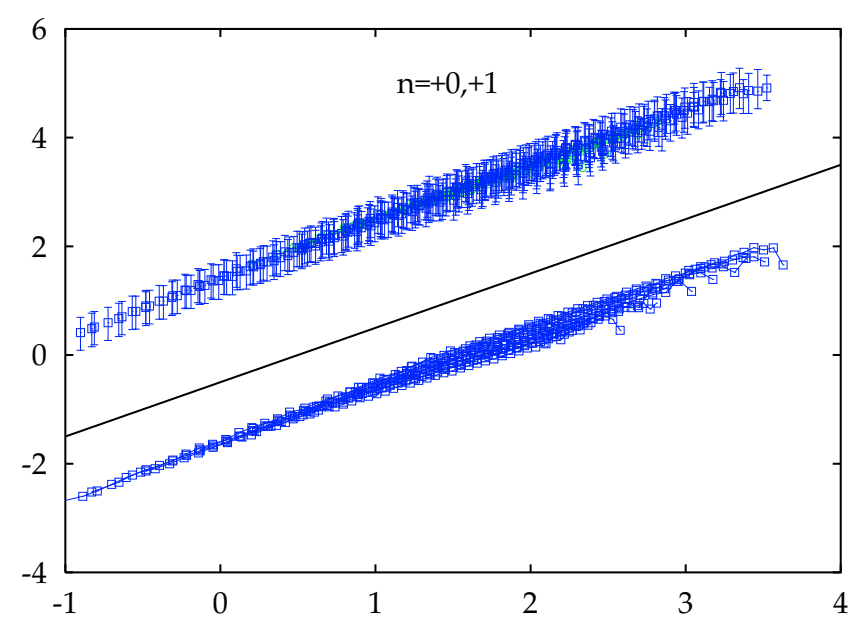

Fig. 7. Abscissa: $\log (1+\delta)$. Ordinate: $\log \left[\kappa_{\mathrm{mac}} / \varrho_{b}\left(\dot{a} r_{\mathrm{nl}}\right)^{2}\right]$. $\left(\kappa_{\mathrm{mac}}\right.$ for the case $n=0$ is shifted by a factor $10^{3}$ ). Solid line: theoretical functional dependence, Eq. (13). Only plotted are the coarsening lengths such that $\sigma(L)>\sqrt{10}$. The scatter bars are barely dependent on time, coarsening length and density; for clarity, they are shown only for $n=0$. 


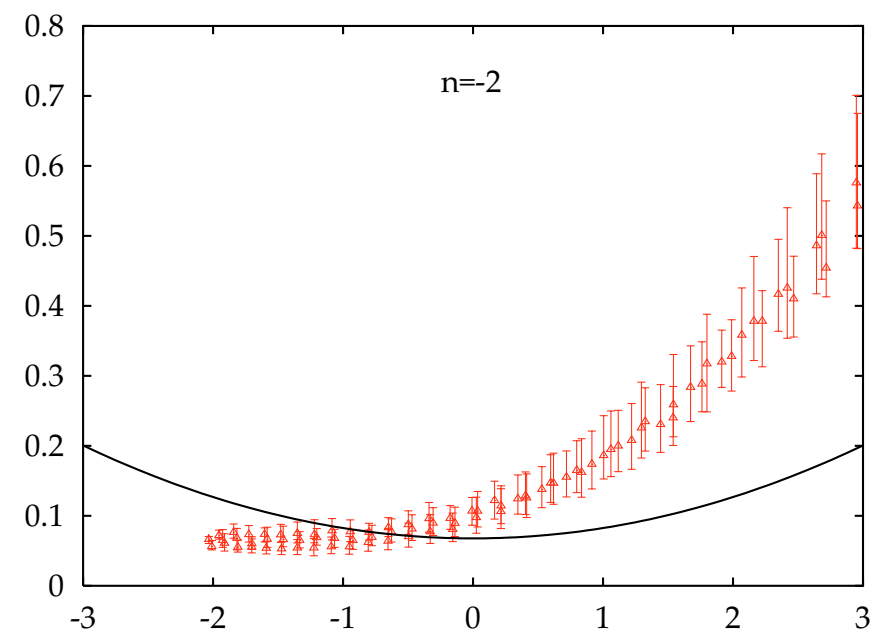

Fig. 8. Abscissa: $\delta / \sigma(L)$. Ordinate: $\kappa_{\text {int }} / \varrho_{b}[\dot{a} L \sigma(L)]^{2}$. Solid line: theoretical prediction, Eq. (6). Only coarsening lengths are plotted such that $\sigma(L)<0.3$.

At the intermediate time, $r_{\mathrm{nl}}=R / 16$, a similar behavior can be detected, although the polytropic-like dependence (15) can be discerned only with some difficulty, Fig. 12. Apparently, the dynamical evolution has not proceeded so far that the intermediate regime with $\eta \neq 0$ can be clearly detected without interference of finite-mass effects: they show up in this case as an artificial reduction of $\kappa_{\text {int }}$ when $\delta$ is small enough, i.e., when there are only a few simulation particles in the coarsening cells, see Fig. 12.

\subsection{CDM models}

Regarding the CDM models, we have investigated only the relationship $\kappa_{\text {int }}(\delta)$ in the nonlinear regime at the present epoch, in order to assess the possibility of a polytropic-like dependence as in the self-similar models. Figure 13 shows the measured $\kappa_{\text {int }}$ as a function of the density for the flat CDM model simulated in a box of sidelength $128 \mathrm{Mpc}$. Now there is no reason to expect an exact scaling behavior like (3) and the plots cannot be made to collapse on a single function. Nevertheless, a dependence $\kappa_{\text {int }} \propto \delta^{2-\eta}$ fits well the data for $\delta$ large enough, with a scale-dependent exponent, $\eta(L)$, which decreases with decreasing $L$ and ranges between 0 and $\approx 0.5$ for the lengths $L$ which we probed ( $L \gtrsim 0.5 \mathrm{Mpc}$, see Sect. 3 ).

Figure 14 shows the measurements of $\kappa_{\text {int }}$ of the flat CDM model in two different simulation boxes (128 and $512 \mathrm{Mpc}$ sidelength, respectively). The worse massresolution in the largest box implies that, for a given $L$ in the highly non-linear regime, the minimum measurable value of $\delta$ is larger. It also means that the absolute value of $\kappa_{\text {int }}$ is smaller. The interesting finding is that, if $\kappa_{\text {int }}$ is multiplied by a factor $\approx 10^{3}$, the plots corresponding to different simulation boxes but to the same coarsening length superpose each other.

The conclusions extracted from the open CDM models are qualitatively identical to those reached with the flat CDM model, and the numerical values for the exponent $\eta$ are very similar.
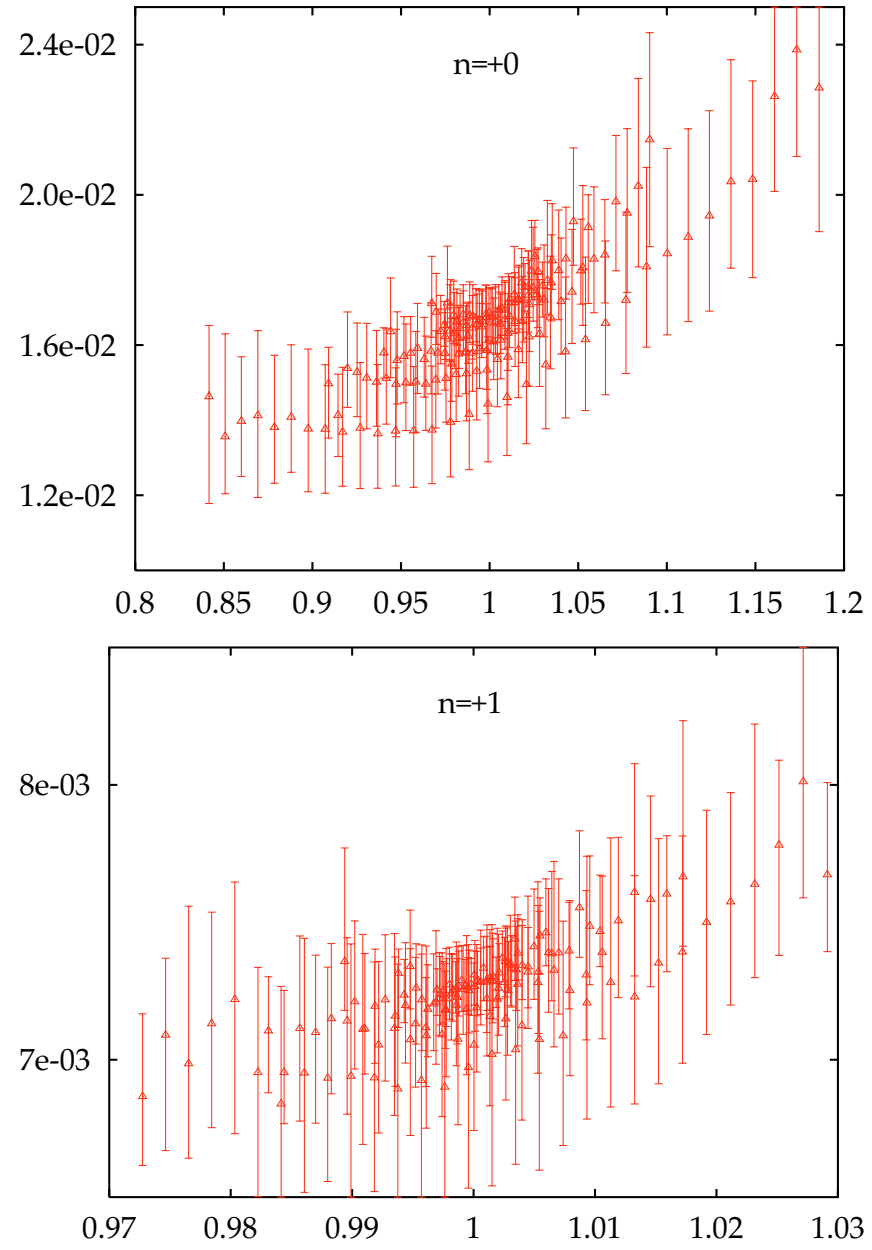

Fig. 9. Abscissa: $1+\delta$. Ordinate: $\kappa_{\text {int }} / \varrho_{b}\left(\dot{a} r_{\mathrm{nl}}\right)^{2}$. Theoretical prediction: a linear dependence. Only coarsening lengths such that $\sigma(L)<0.05$ if $n=0$ and $\sigma(L)<0.03$ if $n=+1$.

\section{Discussion and conclusions}

In the previous section we have measured the macroscopic and the internal kinetic energies of cubic cells as a function of time, cell size and cell mass for different cosmological models. The use of self-similar models simplifies the task of comparing with theoretical results, when available. In particular, the scaling relationship (3) is useful to assess unphysical dependences on the unavoidable additional length scales introduced by the simulation procedure, namely the box sidelength, $R$, and the mean interparticle separation, $\ell$. It must be noticed that, even when the results obey the scaling (3), this does not imply irrelevance of these extra length scales: one can conclude at most that $R$ and $\ell$ could enter in the result solely as the combination $R / \ell$, or equivalently, as $N$, the total particle number. We have not explored explicitly the influence of such a dependence on $N$. Nevertheless, from our results we can obtain some hints about how well they reproduce the limit $N \rightarrow+\infty$.

We first considered the unconstrained averages, $\left\langle K_{\text {int } / \text { mac }}\right\rangle$. We find that $\left\langle K_{\mathrm{mac}}\right\rangle$ for $n=-2$ suffers from strong finitesize effects in a predictable manner. The mean internal kinetic energy, $\left\langle K_{\text {int }}\right\rangle$, however, is strongly affected by resolution effects, the more so the larger $n$ is, and its measurement is 

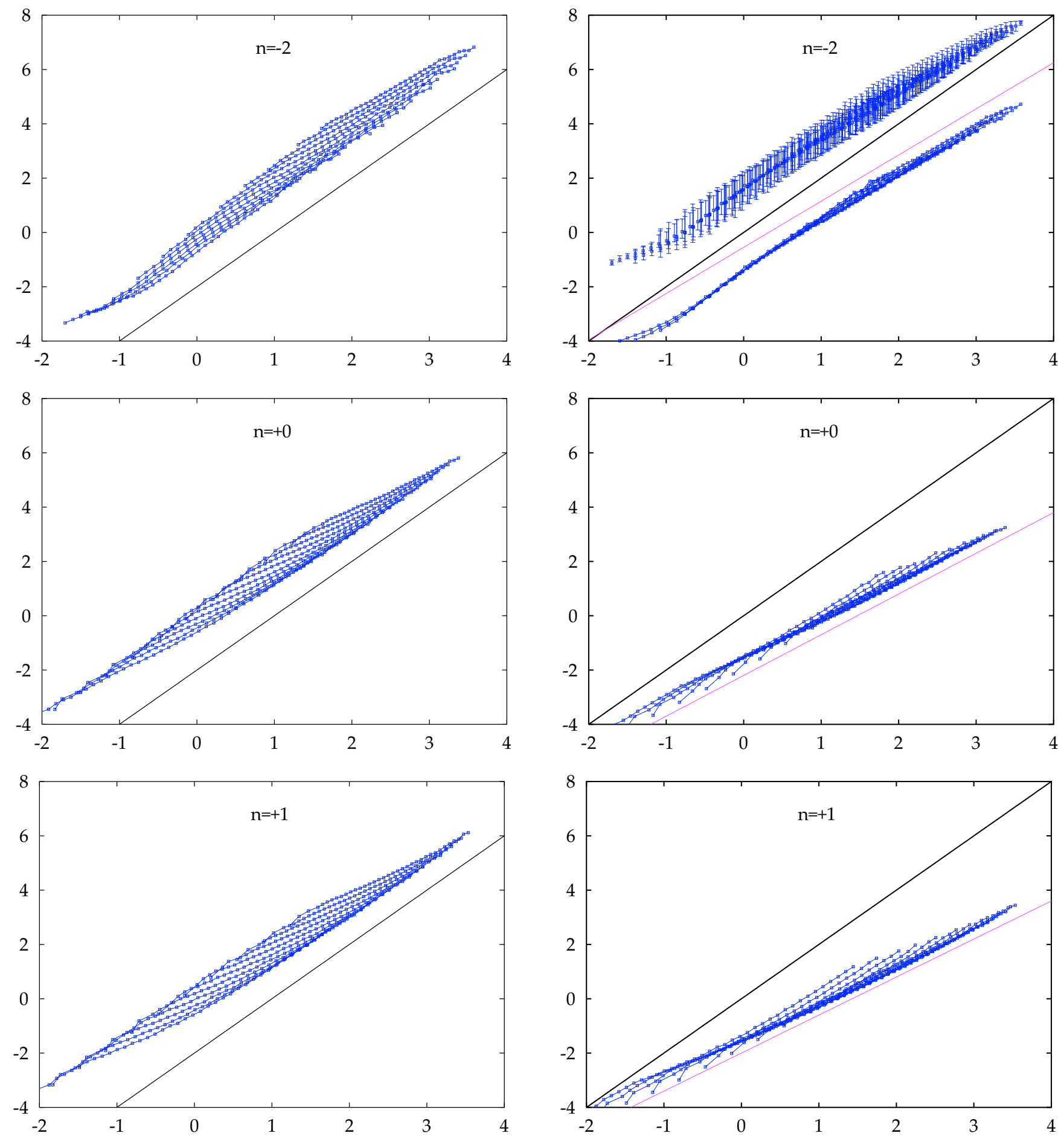

Fig. 10. Abscissa: $\log (1+\delta)$. Ordinate: $\log \left[\kappa_{\text {int }} / \varrho_{b}(\dot{a} L)^{2}\right]$. Solid line: theoretical functional dependence, Eq. (8). Plotted only coarsening lengths at the latest time, $r_{\mathrm{nl}}=R / 4$, such that $\sigma(L)>3$. For clarity, the scatter bars are omitted; see Fig. 11.

therefore unreliable. Next we considered the constrained averages, $\kappa_{\mathrm{int}}, \kappa_{\mathrm{mac}}$. In general, they are much less affected by resolution effects, which are "localized" to very small mass densities or to the earliest time, being more conspicuous for $n=+1$.

The macroscopic kinetic energy, $\kappa_{\mathrm{mac}}$, of the case $n=-2$ depends strongly on $R$, as predicted theoretically. However, this does not break self-similarity of the amplitude of density

Fig. 11. Same as Fig. 10, but now the ordinate is $\log \left[\kappa_{\text {int }} / \varrho_{b}[\dot{a} L \sigma(L)]^{2}\right]$. The solid lines $\left(\kappa_{\text {int }} \propto \delta^{2-\eta}\right)$ have $\eta=0$ and $\eta(n=-2) \approx 0.3, \eta(n=$ $0) \approx 0.5, \eta(n=+1) \approx 0.6$ The case $n=-2$ shows also the same data furnished with scatter bars and shifted by a factor $10^{3}$ for clarity. The scatter bars of the other two cases are of the same size.

fluctuations, as shown by Jain \& Bertschinger $(1996,1998)$, or of $\kappa_{\text {int }}$, as argued in Sect. 2 and exemplified by our results for $\kappa_{\text {int }}$. Relevant for the dynamical evolution of these physical quantities is not the bulk velocity field, but the relative velocity (that is, the velocity gradient), which does not suffer this $R$-dependence. 


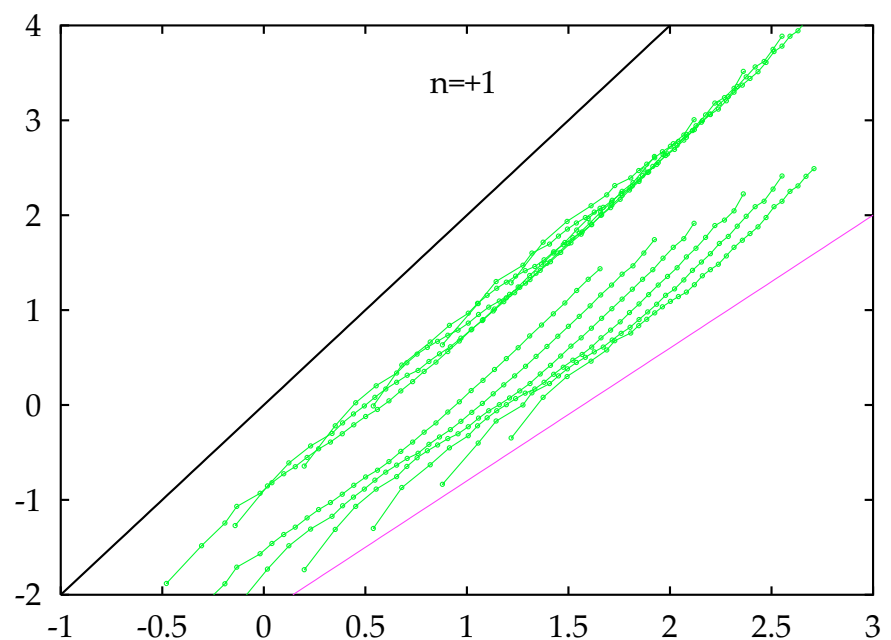

Fig. 12. For $n=+1, r_{\mathrm{nl}}=R / 16, \sigma(L)>3$ : the uppermost data are scaled like in Fig. 10, the lowermost data like in Fig. 11. For the other values of $n$, the behavior of the data is the same.

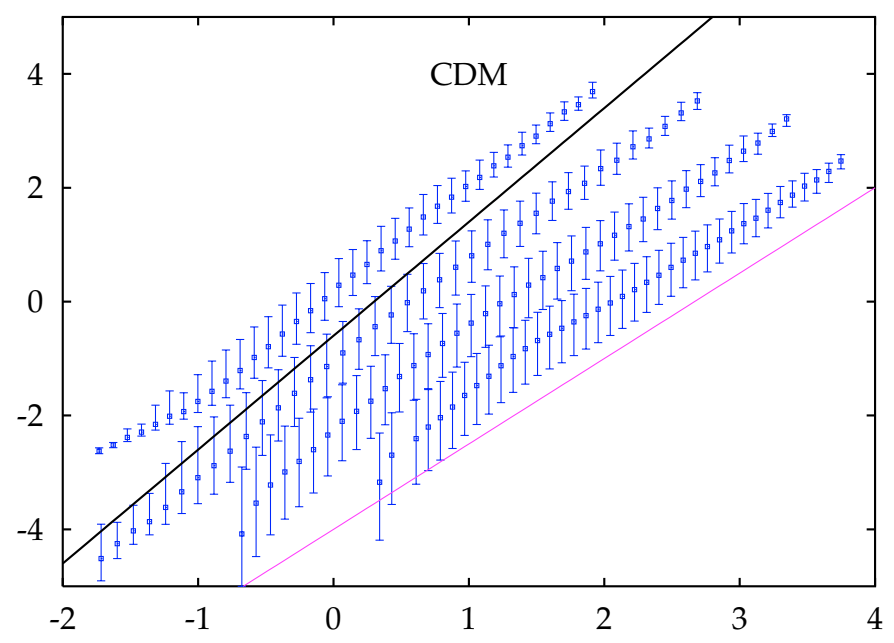

Fig. 13. Abscissa: $\log (1+\delta)$. Ordinate: $\log \kappa_{\text {int }}$ (arbitrary normalization) for the flat CDM model in a box of sidelength $128 \mathrm{Mpc}$. For clarity, only some coarsening lengths are plotted (decreasing top down). The solid lines $\left(\kappa_{\text {int }} \propto \delta^{2-\eta}\right)$ have $\eta=0$ and $\eta=0.5$.

In the cases $n=0,+1$, the theoretical predictions and the scaling (3) are well followed except at the earliest times and smallest cell sizes, when resolution effects are expected to be most important. An interesting result is the linear dependence of $\kappa_{\text {mac }}$ with $\delta$, Eq. (13), with a proportionality factor which according to Fig. 7 does not seem to depend sensitively on the spectral index $n$. Seto \& Sugiyama (2001) have studied $\kappa_{\text {mac }}$ in the cases $n=0,+1$ in the quasilinear regime $(0.2 \lesssim \sigma \lesssim 1.2)$, which we have not addressed at all.

The internal kinetic energy, $\kappa_{\text {int }}$, is more sensitive to the small scale dynamics than $\kappa_{\text {mac }}$ is; correspondingly, resolution effects are found to be more important than for $\kappa_{\text {mac }}$. However, only at the earliest time and smallest coarsening cell sizes for $n=0,+1$ do they render the results unreliable. That's why the theoretical prediction for $\kappa_{\text {int }}(\delta)$ in the linear regime could not be tested when $n=0,+1$; when $n=-2$ the reason is that

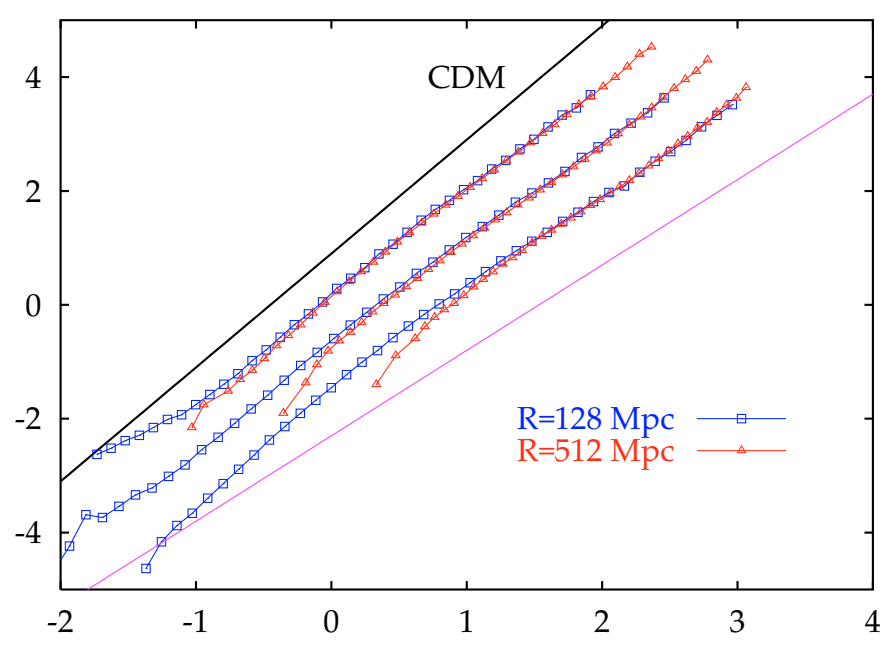

Fig. 14. Abscissa: $\log (1+\delta)$. Ordinate: $\log \kappa_{\text {int }}$ (arbitrary normalization) for the flat CDM model in boxes of sidelength $128 \mathrm{Mpc}$ and $512 \mathrm{Mpc}$, respectively. In the later case, $\kappa_{\text {int }}$ was multiplied by a factor $10^{3}$. For clarity, only some coarsening lengths are plotted. The solid lines $\left(\kappa_{\text {int }} \propto \delta^{2-\eta}\right)$ have $\eta=0$ and $\eta=0.5$.

the linear regime was not really probed, which can be traced back to a finite-size effect.

In the nonlinear regime, the function $\kappa_{\text {int }}(\delta)$ exhibits an interesting behavior. The virial prediction, Eq. (8), was observed only for $n=0,+1$ asymptotically in the large- $\delta$ end of the curves. Otherwise, a polytropic-like dependence (15) was found to fit better the data, with an exponent which does not seem to depend on time or coarsening length, only on the spectral index $n$. The same polytropic-like dependence is found for CDM models, albeit with a scale-dependent exponent, confirming the results of an earlier work (Domínguez 2003). The values of the exponent $\eta$ for the CDM models analyzed here and by Domínguez (2003) are consistent with each other and with those of the self-similar models, in spite of the differences in the number of particles in the simulations (and, compared to Domínguez 2003, the simulation algorithm itself). This suggests that the polytropic-like relation is not an artifact of the simulations, which in this respect seem to reproduce acceptably the limit $N \rightarrow+\infty$. Another hint in this direction is the simple relation which connects the results of the CDM models in boxes of different size, Sect. 4.4: in the larger box, $R=$ $512 \mathrm{Mpc}$, a coarsening cell of a given mass contains less particles that in the smaller box, $R=128 \mathrm{Mpc}$. Nevertheless, this mass-resolution effect does not alter the functional dependence $\kappa_{\text {int }}(\delta)$ at all, and can be accounted for by a scaleindependent constant offset.

In the work by Kepner et al. (1997), $\kappa_{\text {int }}$ is also measured in CDM simulations (in their notation, $\kappa_{\text {int }}=\varrho \sigma_{v}^{2} / 2$ ). They find a polytropic-like dependence too, but with a slightly smaller exponent, $\eta \approx 0.1$ (Kepner et al. 1997, Eqs. (17)-(18)). We believe this discrepancy to be a consequence of their simulation having too few particles $\left(N=32^{3}\right)$ : as a consequence, they measured the function $\kappa_{\text {int }}(\delta)$ with coarsening cells having at most 100 particles (in one case); in many cases, the cells have less than a few tens of particles (Kepner et al. 1997, Fig. 3). For comparison, the polytropic-like dependence in Fig. 13 is 
detected in coarsening cells containing a number of particles spanning ranges as wide as 30-7000 or 500-30000 (see also Domínguez 2003). The work by Kepner et al. (1997) was motivated by comparison with redshift surveys. It relied on the cosmic virial theorem and particular emphasis was put on the dependence with cosmological parameters $\left(\Omega_{m}, \Omega_{\Lambda}\right)$. Our results show that departures from the virial prediction are not small at all, so that the method devised by Kepner et al. (1997) must be adjusted. More generally, our results warn against a straightforward use of the cosmic virial theorem to estimate cosmological parameters from observations without first assessing that the employed observational data do indeed pertain virialized structures.

In the work by Nagamine et al. (2001), the cosmic Mach number $\left(=K_{\mathrm{mac}} / K_{\mathrm{int}}\right.$ in our notation, and $\kappa_{\mathrm{int}}=\varrho \sigma^{2} / 2$ in theirs) is measured in a $\Lambda \mathrm{CDM}$ hydrodynamical simulation, for three different length scales and as a function of the density. As a side-result, they also find a polytropic-like dependence for the velocity dispersion of groups of DM halos and galaxies (with $\eta \approx 0.5-0.7$ ) - the authors do not elaborate much on this result. One must keep in mind that, compared to our simulations, theirs involves also the baryonic component and the formation of galaxies, which can affect the velocity dispersion (Tissera \& Domínguez-Tenreiro 1998).

One can conceive two natural extreme cases of a polytropic-like dependence: the "virial" case, $\eta_{\text {virial }}=0$, when velocity dispersion is fixed by the local mass density, and the "isothermal" case, $\eta_{\text {isothermal }}=1$, when velocity dispersion is fixed by an external cause, e.g. tidal forces, free flow,... The values of $\eta$ that we measure invariably fall between 0 and 1 ; the corresponding relations $\kappa_{\text {int }}(\delta)$ can be arguably understood as the outcome of the competition of the two effects ("local virialization vs. global thermalization"), whose relative strength varies with the spectral index $n$ and the cell size and mass. However, an elaborated theory is required to lend support to this explanation, the ultimate goal being the "postdiction" of the relation (15). The value $\eta=1 / 3$ was derived theoretically by Buchert \& Domínguez (1998), but we think this is irrelevant to our results, since certain restrictive assumptions were made (vanishingly small and isotropic velocity dispersion, approximately shear-free velocity field $\boldsymbol{u}$ ), which are unlikely to hold in the regime where we find the polytropic-like dependence. The results from the simulations cannot be explained by any theory whose starting point is the usual thermodynamical theory or, more generally, the (grand-)canonical ensemble of statistical mechanics (Saslaw \& Fang 1996; Hochberg \& Pérez-Mercader 1996; de Vega et al. 1998), since in that framework the kinetic energy is an extensive variable: $\kappa_{\mathrm{int}}=T \varrho(T$ is the kinetic temperature) and $\eta=1$. As a side-remark, we notice that Saslaw et al. (1990) compute the velocity distribution allegedly in the framework of thermodynamics: but they use contradictory arguments and obtain instead that the kinetic energy scales like $\varrho^{2}$ (that is, $\eta=0$ ), and a velocity distribution different from the Maxwellian one characteristic of thermal equilibrium and which should follow from the (grand-)canonical ensemble probability.

The discovered relationship $\kappa_{\text {int }}(\varrho)$ is useful for an improved model of structure formation by gravitational instability
(Buchert \& Domínguez 1998): the dust model (pressureless fluid) is added a term proportional to the gradient of $\kappa_{\text {int }}$ (a kinetic pressure), in order to account for the reaction of the dynamically generated velocity dispersion on the evolution. The evolution equation for the velocity field $\boldsymbol{u}(\boldsymbol{x}, t)$ then reads

$$
\frac{\partial \boldsymbol{u}}{\partial t}=-\frac{\dot{a}}{a} \boldsymbol{u}-\frac{1}{a}(\boldsymbol{u} \cdot \nabla) \boldsymbol{u}+\boldsymbol{w}-\frac{1}{a \varrho} \nabla \kappa_{\mathrm{int}},
$$

where the peculiar gravitational acceleration $w$ is given by Poisson's equation. Further theoretical studies of this model (Adler \& Buchert 1999; Buchert et al. 1999; Maartens et al. 1999; Morita \& Tatekawa 2001; Tatekawa et al. 2002) work with a $\kappa_{\text {int }}$ which depends only on density, e.g. a pure polytropic-like dependence with values of the exponent $\eta$ in concordance with our measurements. Our results show that the functional dependence of $\kappa_{\text {int }}$ on $\varrho$ is somewhat more complicated than purely polytropic and changes with time and coarsening length. Nevertheless, since the term $-\nabla \kappa_{\text {int }}$ is a pressure, it opposes compression in collapsing regions. More can be learned about the behavior of this term when some simplifications are introduced (Buchert \& Domínguez 1998; Buchert et al. 1999): one assumes that the evolution follows the dust model prediction in the form of the Zel'dovich approximation (basically that $\boldsymbol{u} \propto \boldsymbol{w}$ ) "almost everywhere", i.e., except near potential density singularities where the effect of $-\nabla \kappa_{\text {int }}$ becomes relevant. One can then apply boundary-layer theory to show (Domínguez 2000) that this term does behave "adhesively" and indeed succeeds in preventing the formation of a singularity, provided $\kappa_{\text {int }}$ is a function of $\varrho$ and $\kappa_{\text {int }}(\varrho) / \varrho$ is a growing function of $\varrho$ (meaning $\eta<1$ for a polytropiclike dependence). This behavior is robust against the observed time-dependence in the relation $\kappa_{\text {int }}(\varrho)$, being much slower than the time-scale of collapse. One can conclude that the dependence $\kappa_{\text {int }}(\varrho ; t)$ measured in $N$-body simulations leads to the same qualitative "adhesive" behavior as the simpler dependences addressed theoretically in the literature.

When it comes to inserting our results in the theoretical model (16), there are some issues which we have not addressed but may be relevant to a better understanding of the model. First, it must be noticed that the average relationship $\kappa_{\text {int }}(\varrho)$ does not mean in principle a one-to-one dependence between $K_{\text {int }}$ and $\varrho$; on the contrary, the data scatter around the average dependence, Fig. 11. In fact, the derivation of Eq. (16) yields in reality a term $-\nabla K_{\text {int }}$ (Buchert \& Domínguez 1998): the influence of the scatter on the model outputs should be quantified and, if proven relevant, incorporated in the model, e.g. as a noisy source (Buchert et al. 1999). Another issue of possible concern is the amount of velocity dispersion in the coarsening cells associated to "bound structures" (as opposed to the amount associated to particle flow between neighboring cells); in this context, it would also be interesting to assess the contribution to velocity dispersion from "ordered motion", e.g. due to a net angular momentum.

In conclusion, we have studied the density dependence of the macroscopic and internal kinetic energies in coarsening cells. We could identify the influence of finite-size and resolution effects on the measured physical quantities. When these effects were irrelevant, we could confirm some of the 
theoretical asymptotic predictions. Finally, we found that in an intermediate range of densities, the velocity dispersion scales as a power of the mass density, with an exponent different from the virial prediction.

Acknowledgements. A.D. acknowledges support of the "Sonderforschungsbereich SFB 375 für Astro-Teilchenphysik der Deutschen Forschungsgemeinschaft". A.L.M. acknowledges support of US NSF through grant AST0070702 and the National Center for Supercomputing Applications (Urbana, Illinois, USA).

\section{Appendix A: Estimates with the linear solution}

In this Appendix we collect the mathematical calculations which lead to Eqs. (4)-(6). The main idea is that $\kappa_{\text {int }}$ and $\kappa_{\text {mac }}$ are determined by the dominant contribution of modes in the linear regime when $L \gg r_{\mathrm{nl}}$, so that they can be estimated by inserting the linear solution in the definitions (1). These definitions can be rewritten as follows:

$\varrho(x, t)=\int \frac{\mathrm{d} \boldsymbol{y}}{L^{3}} W\left(\frac{\boldsymbol{x}-\boldsymbol{y}}{L}\right) \varrho_{\text {mic }}(\boldsymbol{y}, t)$,

$\varrho \boldsymbol{u}(\boldsymbol{x}, t)=\int \frac{\mathrm{d} \boldsymbol{y}}{L^{3}} W\left(\frac{\boldsymbol{x}-\boldsymbol{y}}{L}\right) \varrho_{\mathrm{mic}} \boldsymbol{u}_{\mathrm{mic}}(\boldsymbol{y}, t)$,

$K_{\mathrm{int}}(\boldsymbol{x}, t)=\frac{1}{2} \int \frac{\mathrm{d} \boldsymbol{y}}{L^{3}} W\left(\frac{\boldsymbol{x}-\boldsymbol{y}}{L}\right) \varrho_{\mathrm{mic}} \boldsymbol{u}_{\mathrm{mic}}^{2}(\boldsymbol{y}, t)-\frac{1}{2} \varrho \boldsymbol{u}^{2}(\boldsymbol{x}, t)$,

in terms of the formal microscopic fields ${ }^{4}$

$\varrho_{\text {mic }}(\boldsymbol{x}, t):=\frac{m}{a(t)^{3}} \sum_{\alpha} \delta_{\mathrm{D}}\left(\boldsymbol{x}-\boldsymbol{x}_{\alpha}(t)\right)$

$\varrho_{\text {mic }} \boldsymbol{u}_{\text {mic }}(\boldsymbol{x}, t):=\frac{m}{a(t)^{3}} \sum_{\alpha} \boldsymbol{u}_{\alpha}(t) \delta_{\mathrm{D}}\left(\boldsymbol{x}-\boldsymbol{x}_{\alpha}(t)\right)$.

We introduce the Fourier transform of any spatial field $\phi(\mathbf{x})$, denoted by a tilde and defined as

$\tilde{\phi}(\boldsymbol{k}):=\int \mathrm{d} \boldsymbol{x} \mathrm{e}^{\mathrm{i} \boldsymbol{k} \cdot \boldsymbol{x}} \phi(\boldsymbol{x})$.

The velocity then reads

$\varrho \boldsymbol{u}(\boldsymbol{x}, t)=\int \frac{\mathrm{d} \boldsymbol{k} \mathrm{d} \boldsymbol{q}}{(2 \pi)^{6}} \mathrm{e}^{-\mathrm{i}(\boldsymbol{k}+\boldsymbol{q}) \cdot \boldsymbol{x}} \tilde{\varrho}_{\text {mic }}(\boldsymbol{k}, t) \tilde{\boldsymbol{u}}_{\text {mic }}(\boldsymbol{q}, t) \tilde{W}(L(\boldsymbol{k}+\boldsymbol{q}))$,

and the internal kinetic energy

$K_{\text {int }}(\boldsymbol{x}, t)=\frac{1}{2} \int \frac{\mathrm{d} \boldsymbol{k} \mathrm{d} \boldsymbol{q} \mathrm{d} \boldsymbol{p}}{(2 \pi)^{9}} \mathrm{e}^{-\mathrm{i}(\boldsymbol{k}+\boldsymbol{q}+\boldsymbol{p}) \cdot \boldsymbol{x}} \tilde{\varrho}_{\text {mic }}(\boldsymbol{k}, t)$

$\times\left[\tilde{\boldsymbol{u}}_{\mathrm{mic}}(\boldsymbol{q}, t) \cdot \tilde{\boldsymbol{u}}_{\mathrm{mic}}(\boldsymbol{p}, t) \tilde{W}(L(\boldsymbol{k}+\boldsymbol{q}+\boldsymbol{p}))-\tilde{\boldsymbol{u}}(\boldsymbol{q}, t) \cdot \tilde{\boldsymbol{u}}(\boldsymbol{p}, t) \tilde{W}(L \boldsymbol{k})\right]$.

Even if $L \gg r_{\mathrm{nl}}$, these expressions contain contributions from nonlinear modes $\left(r_{\mathrm{nl}} k \gg 1\right)$. The hypothesis (Sect. 2) is that these contributions are nevertheless negligible compared to those from the linear modes $\left(r_{\mathrm{nl}} k \ll 1\right)$. This implies

\footnotetext{
${ }^{4}$ In the mathematical manipulations in this Appendix, one can assume that the Dirac delta is regularized by a short-distance cutoff which is set to zero in the final results. In this way, the field $\boldsymbol{u}_{\text {mic }}(\boldsymbol{x})$ is defined, and not only the product $\varrho_{\text {mic }} \boldsymbol{u}_{\text {mic }}(\boldsymbol{x})$.
}

$\tilde{\boldsymbol{u}}(\boldsymbol{k}) \approx \tilde{\boldsymbol{u}}_{\text {mic }}(\boldsymbol{k}) \tilde{W}(L \boldsymbol{k})$ in the linear regime, and the growing linear solution in an Einstein-de Sitter background yields in turn

$\tilde{\boldsymbol{u}}_{\text {mic }}(\boldsymbol{k}, t) \approx-\dot{\mathrm{a}} \frac{\mathrm{k}}{k^{2}} \tilde{\delta}_{\text {mic }}(\boldsymbol{k}, t), \quad\left(r_{\mathrm{nl}} k \ll 1\right)$

with $\delta_{\text {mic }}=\left(\varrho_{\text {mic }} / \varrho_{b}\right)-1$. Inserting these linear relations in the definition (A.1) we obtain, to lowest order in the inhomogeneities,

$$
\begin{aligned}
& K_{\mathrm{int}}(\boldsymbol{x}, t) \approx \frac{1}{2} \varrho_{b} \dot{a}^{2} \int \frac{\mathrm{d} \boldsymbol{k} \mathrm{d} \boldsymbol{q}}{(2 \pi)^{6}} \mathrm{e}^{-\mathrm{i}(\boldsymbol{k}+\boldsymbol{q}) \cdot \boldsymbol{x}} \frac{\boldsymbol{k} \cdot \boldsymbol{q}}{k^{2} q^{2}} \\
& \times[\tilde{W}(L \boldsymbol{k}) \tilde{W}(L \boldsymbol{q})-\tilde{W}(L(\boldsymbol{k}+\boldsymbol{q}))] \tilde{\delta}_{\mathrm{mic}}(\boldsymbol{k}, t) \tilde{\delta}_{\mathrm{mic}}(\boldsymbol{q}, t) .
\end{aligned}
$$

For Gaussian initial conditions, the average of Eq. (2) can be easily computed using standard techniques for the Gaussian functional integrals (e.g. Zinn-Justin 1996):

$$
\begin{aligned}
& \left\langle\delta_{\mathrm{D}}(\varrho(\boldsymbol{x}, t ; L)-\varrho)\right\rangle=\frac{1}{\sqrt{2 \pi} \varrho_{b}(t) \sigma(L, t)} \exp \left(-\frac{\delta^{2}}{2 \sigma^{2}(L, t)}\right), \\
& \frac{\left\langle\tilde{\delta}_{\text {mic }}(\boldsymbol{k}, t) \tilde{\delta}_{\text {mic }}(\boldsymbol{q}, t) \delta_{\mathrm{D}}(\varrho(\boldsymbol{x}, t ; L)-\varrho)\right\rangle}{\left\langle\delta_{\mathrm{D}}(\varrho(\boldsymbol{x}, t ; L)-\varrho)\right\rangle}=(2 \pi)^{3} P(k, t) \delta_{\mathrm{D}}(\boldsymbol{k}+\boldsymbol{q}) \\
& -\frac{1}{\sigma^{2}(L, t)}\left(1-\frac{\delta^{2}}{\sigma^{2}(L, t)}\right) P(k, t) P(q, t) \tilde{W}(L \boldsymbol{k}) \tilde{W}(L \boldsymbol{q}) \mathrm{e}^{\mathrm{i}(\boldsymbol{k}+\boldsymbol{q}) \cdot \boldsymbol{x}} .
\end{aligned}
$$

With these expressions, one recovers the result (6) with the coefficients given by

$$
\begin{aligned}
\left\langle K_{\text {int }}\right\rangle(L, t) & =\frac{1}{2} \varrho_{b} \dot{a}^{2} \int \frac{\mathrm{d} \boldsymbol{k}}{(2 \pi)^{3}} \frac{P(k)}{k^{2}}\left[1-|\tilde{W}(L \boldsymbol{k})|^{2}\right], \\
B(L, t)= & \frac{\varrho_{b} \dot{a}^{2}}{2 \sigma^{2}\left\langle K_{\mathrm{int}}\right\rangle(L, t)} \int \frac{\mathrm{d} \boldsymbol{k} \mathrm{d} \boldsymbol{q}}{(2 \pi)^{6}} \frac{\boldsymbol{k} \cdot \boldsymbol{q}}{k^{2} q^{2}} \\
& \times P(k, t) P(q, t) \tilde{W}(L \boldsymbol{k}) \tilde{W}(L \boldsymbol{q}) \tilde{W}(L(\boldsymbol{k}+\boldsymbol{q})) .
\end{aligned}
$$

These integrals are IR-convergent provided $P(k \rightarrow 0) \sim$ $k^{n},-3<n$, since $\tilde{W}(\boldsymbol{k} \rightarrow \mathbf{0}) \sim 1+o\left(k^{2}\right)$ ( $\leftrightarrow$ the window function is normalized to unity and decays fast enough at large distances). On the other hand, there is an implicit ultraviolet (UV) cutoff, $k, q \leq r_{\mathrm{nl}}^{-1}$, because these expressions have been derived for the linear regime. The limit " $r_{\mathrm{nl}} \rightarrow 0$ " of the coefficient $B$ is finite, since we assume that $\tilde{W}(\boldsymbol{k} \rightarrow \infty) \rightarrow 0$ fast enough ( $\leftrightarrow$ the window is smooth enough: the decay is exponential for a Gaussian window, algebraic $\left(\sim k^{-2} \cos k\right)$ for a spherical top-hat window). $\left\langle K_{\text {int }}\right\rangle$ is however UV-convergent only if $P(k \rightarrow \infty) \sim k^{n}, n<-1$, indicating that it is determined by nonlinear modes when $n>-1$.

A similar reasoning can be repeated for the macroscopic kinetic energy: inserting the linear relationships in the definition $K_{\text {mac }}=(1 / 2) \varrho u^{2}$ one gets

$\kappa_{\mathrm{mac}} \approx \frac{1}{2} \varrho_{b} \dot{a}^{2} \int \frac{\mathrm{d} \boldsymbol{k}}{(2 \pi)^{3}} \frac{P(k)}{k^{2}}|\tilde{W}(L \boldsymbol{k})|^{2}=\left\langle K_{\mathrm{mac}}\right\rangle$,

which is IR-divergent in the range of spectral indices $-3<n<-1$. 


\section{References}

Adler, S., \& Buchert, T. 1999, A\&A, 343, 317

Bernardeau, F., Colombi, S., Gaztañaga, E., \& Scoccimarro, R. 2002, Phys. Rep., 367, 1

Buchert, T., \& Domínguez, A. 1998, A\&A, 335, 395

Buchert, T., Domínguez, A., \& Pérez-Mercader, J. 1999, A\&A, 349, 343

de Vega, H., Sánchez, N., \& Combes, F. 1998, ApJ, 500, 8

Dekel, A. 1994, ARA\&A, 32, 371

Domínguez, A. 1999, Ph.D. Thesis, Univ. Autónoma de Madrid

Domínguez, A. 2000, Phys. Rev. D, 62, 103501

Domínguez, A. 2002, MNRAS, 334, 435

Domínguez, A. 2003, Astron. Nachr., 324, 560

Feldman, H., Juszkiewicz, R., Ferreira, P., et al. 2003, ApJ, 596, L131

Gurbatov, S. N., Saichev, A. I., \& Shandarin, S. F. 1989, MNRAS, 236, 385

Hochberg, D., \& Pérez-Mercader, J. 1996, Gen. Rel. Grav., 28, 1427

Jain, B., \& Bertschinger, E. 1996, ApJ, 456, 43

Jain, B., \& Bertschinger, E. 1998, ApJ, 509, 517

Kepner, J. V., Summers, F. J., \& Strauss, M. A. 1997, New Astron., 2, 165

Klypin, A., \& Holtzmann, J. 1997 [arXiv: astro-ph/9712217]

Knebe, A., \& Müller, V. 1999, A\&A, 341, 1

Kofman, L., Pogosyan, D., Shandarin, S. F., \& Melott, A. L. 1992, ApJ, 393, 437

Kofman, L., \& Shandarin, S. 1988, Nature, 334, 129
Maartens, R., Triginer, J., \& Matravers, D. R. 1999, Phys. Rev. D, 60, 103503

Melott, A. L., \& Shandarin, S. F. 1993, ApJ, 410, 469

Melott, A. L., Shandarin, S. F., \& Weinberg, D. H. 1994, ApJ, 428, 28

Morita, M., \& Tatekawa, T. 2001, MNRAS, 328, 815

Nagamine, K., Ostriker, J. P., \& Cen, R. 2001, ApJ, 553, 513

Ostriker, J. P., \& Suto, Y. 1990, ApJ, 348, 378

Padmanabhan, T. 1995, Structure Formation in the Universe (Cambridge Univ. Press)

Peebles, P. J. E. 1980, The Large-Scale Structure of the Universe (Princeton Univ. Press)

Sahni, V., \& Coles, P. 1995, Phys. Rep., 262, 1

Saslaw, W. C., Chitre, S. M., Itoh, M., \& Inagaki, S. 1990, ApJ, 365, 419

Saslaw, W. C., \& Fang, F. 1996, ApJ, 460, 16

Sathyaprakash, B. S., Sahni, V., Munshi, D., Pogosyan, D., \& Melott, A. L. 1995 , MNRAS, 275,463

Seto, N., \& Sugiyama, N. 2001, ApJ, 558, 505

Strauss, M. A., Ostriker, J. P., \& Cen, R. 1998, ApJ, 494, 20

Tatekawa, T., Suda, M., Maeda, K., Morita, M., \& Anzai, H. 2002, Phys. Rev. D, 66, 064014

Tissera, P. B., \& Domínguez-Tenreiro, R. 1998, MNRAS, 297, 177

Watkins, R., Feldman, H. A., Chambers, S. W., Gorman, P., \& Melott, A. L. 2002, ApJ, 564, 534

Zinn-Justin, J. 1996, Quantum field theory and critical phenomena (Oxford: Clarendon Press) 